\title{
Blow Up and Exponential Growth to a Petrovsky Equation with Degenerate Damping
}

\author{
Fatma Ekinci ${ }^{1}$ and Erhan Pişkin ${ }^{{ }^{*}}$ \\ ${ }^{1}$ Dicle University, Department of Mathematics, 21280 Diyarbakır, Turkey \\ * Corresponding Author
}

\section{Article Info}

Keywords: Blow up, Exponential growth, Petrovsky equation, Degenerate damping.

2010 AMS: 35B40, 35B44, 35G61,

$35 L 75$.

Received: 10 May 2021

Accepted: 27 June 2021

Available online: 30 June 2021

\section{Abstract}

This paper deals with the initial boundary value problem of Petrovsky type equation with degenerate damping. Under some appropriate conditions, we study the finite time blow up and exponential growth of solutions with negative initial energy.

\section{Introduction}

We investigate the following initial boundary value problem:

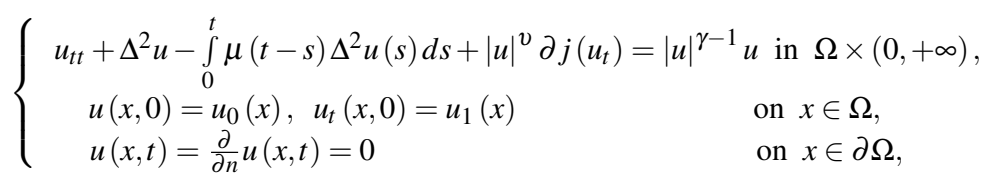

where $\partial j(s)$ denotes the sub-differential of $j(s)$ [1], $n$ is the outer normal and $\Omega$ is a bounded domain in $R^{n}$ with a smooth boundary $\partial \Omega$. The Petrovsky type equations are orginated from the study of beams and plates and so often arise in many branches of physics such as optics, nuclear physics, geophysics and ocean acoustics. Rivera et al. [2] considered

$$
u_{t t}+\Delta^{2} u-\int_{0}^{t} \mu(t-s) \Delta^{2} u(s) d s-\gamma \Delta u_{t t}=0
$$

and proved the asymptotic behaviour of solution with the initial and dynamical boundary conditions.

The following problem was studied by Alabau-Boussouira et al. [3]

$$
u_{t t}+\Delta^{2} u-\int_{0}^{t} \mu(t-s) \Delta^{2} u(s) d s=f(u) .
$$

The authors studied exponential and polynomial decay results of solutions when the memory $\mu$ decay exponentially and polynomially, respectively. Afterwards, Tahamtani ve Shahrouzi [4] investigated the existence of weak solutions for problem (1.2). In addition, the authors proved blow up of solutions with positive and negative initial energy in finite time.

In [5], Li and Gao discussed the following equation

$$
u_{t t}+\Delta^{2} u-\int_{0}^{t} \mu(t-s) \Delta^{2} u(s) d s+\left|u_{t}\right|^{p-2} u_{t}=|u|^{\gamma-2} u .
$$


The authors studied blow up result of solutions under suitable conditions of the initial datum and the relaxation function. Furthermore, problem (1.3) has been studied by Liu et al. [6] and the finite time blow-up of solutions with arbitrary high initial energy has been proved. Recently, Liu et al. [7] investigated problem (1.3) with case $(p=2)$ and proved blow up of solution with $E(0) \leq M, M$ is positive constant. Furthermore, the authors studied blow up of solutions with $E(0)>M$ by applying concavity method.

On the other hand, Messaoudi [8] investigated the following problem

$$
u_{t t}+\Delta^{2} u+\left|u_{t}\right|^{p-2} u_{t}=|u|^{\gamma-2} u .
$$

The author studied an existence result and global solution in case $p \geq \gamma$. Then, blow-up of solutions with negative initial energy and $p<\gamma$ was proved. Then, Chen and Zhou [9] discussed blow up with positive initial energy for (1.4) and showed that the solution blows up in finite time for vanishing initial energy case $(p=2)$. Moreover, the problem (1.4) with $\Delta u_{t}$ term has been considered by Pişkin and Polat [10] and the authors proved decay estimates of the solution by using Nakao's inequality. Some other studies on Petrovsky equations are [11], [12], [13], [14].

The hyperbolic models with degenerate damping also are of much interest in material science and physics. It particularly appears in physics when the friction is modulated by the strains. There are a lot of studies that has degenerate damping terms, namely $\delta(u) h\left(u_{t}\right)$ here $\delta(u)$ is a positive function and $h$ is nonlinear, (see [15-21]).

Motivated by previous results, we prove several results concerning the blow up and exponential growth of solution for the problem (1.1). It should be noted here that we can say that the study is both quite difficult and interesting and the analysis are more subtle because of the degenerate damping.

The remaining part of this paper is organized as follows: In the next section, we study the nonexistence of solutions. The exponential growth result is presented in Section 3.

\section{Preliminaries}

Now, we present some preliminary material which will be helpful in the proof of our results. Throughout this paper, we denote the standart $L^{2}(\Omega)$ norm by $\|\cdot\|=\|\cdot\|_{L^{2}(\Omega)}$ and $L^{q}(\Omega)$ norm $\|\cdot\|_{q}=\|\cdot\|_{L^{q}(\Omega)}$.

(A1) $v, p \geq 0, \gamma>1 ; v \leq \frac{n}{n-2}, \gamma+1 \leq \frac{2 n}{n-2}$ if $n \geq 3$. There exist positive constants $c, c_{0}, c_{1}$ such that for all $s, k \in R j(s): R \rightarrow R$ be a $C^{1}$ convex real function satisfies

- $j(s) \geq c|s|^{p+1}$,

- $j^{\prime}(s)$ is single valued and $\left|j^{\prime}(s)\right| \leq c_{0}|s|^{p}$

- $\left(j^{\prime}(s)-j^{\prime}(k)\right)(s-k) \geq c_{1}|s-k|^{p+1}$.

(A2) $u_{0}(x) \in H_{0}^{2}(\Omega), u_{1}(x) \in L^{2}(\Omega)$.

(A3) Assume $\mu(\tau): R^{+} \rightarrow R^{+}$satisfies

$$
\mu(\tau) \geq 0, \mu^{\prime}(\tau) \leq 0,
$$

for all $s \in R^{+}$and

$$
\int_{0}^{t} \mu(\tau) d \tau<1
$$

(A4) $\int_{0}^{t} \mu(\tau) d \tau<\frac{\gamma-1}{\gamma+1}$.

We use the following notations

$$
\begin{aligned}
l & =1-\int_{0}^{t} \mu(\tau) d \tau, \\
(\mu \diamond \theta)(t) & =\int_{0}^{t} \mu(t-\tau) \int_{\Omega}|\theta(t)-\theta(\tau)| d x d \tau .
\end{aligned}
$$

The said solution of (1.1) satisfies the energy identity

$$
E(t)+\frac{1}{2} \mu(t)\|\Delta u\|^{2}-\frac{1}{2}\left(\mu^{\prime} \diamond \Delta u\right)(t)+\int_{0}^{t} \int_{\Omega}|u(\tau)|^{v} j\left(u_{t}\right)(\tau) d x d \tau=E(0),
$$

where

$$
E(t)=\frac{1}{2}\left[\left\|u_{t}\right\|^{2}+\left(1-\int_{0}^{t} \mu(s) d s\right)\|\Delta u\|^{2}+(\mu \diamond \Delta u)(t)\right]-\frac{1}{\gamma+1}\|u\|_{\gamma+1}^{\gamma+1}
$$

and

$$
E(0)=\frac{1}{2}\left[\left\|u_{1}\right\|^{2}+\left\|\Delta u_{0}\right\|^{2}\right]-\frac{1}{\gamma+1}\left\|u_{0}\right\|_{\gamma+1}^{\gamma+1}
$$

Moreover, by computation, we get

$$
E(t) \leq E(0)
$$




\section{Blow up}

In this section, we shall prove the blow up results of the solutions for problem (1.1).

Theorem 3.1. Let (A1)-(A4) hold. Assume further that $\gamma>v+p, E(0)<0$ and $u$ be a any solution to (1.1) on the interval $[0, T]$, then $T$ is necessarily finite, i.e. u can't be continued for all $t>0$.

Proof. We assume that the solution exists for all time and we arrive to a contradiction. Set

$$
H(t)=-E(t)
$$

By using (2.1), we get

$$
\begin{aligned}
H^{\prime}(t) & =-E^{\prime}(t) \\
& =\frac{1}{2} \mu(t) \|\left.\Delta u\right|^{2}-\frac{1}{2}\left(\mu^{\prime} \diamond \Delta u\right)(t)+\int_{\Omega}|u(t)|^{v} j\left(u_{t}\right) u_{t} d x \\
& \geq \int_{\Omega}|u(t)|^{v} j\left(u_{t}\right) u_{t} d x \\
& \geq c_{0} \int_{\Omega}|u(t)|^{v}\left|u_{t}\right|^{p+1} d x .
\end{aligned}
$$

Hence, we find

$$
0<H(0) \leq H(t) \leq \frac{1}{\gamma+1}\|u\|_{\gamma+1}^{\gamma+1}, \quad t \geq 0
$$

Define

$$
K(t)=H^{1-\rho}(t)+\varepsilon \int_{\Omega} u u_{t} d x
$$

where $\rho=\min \left\{\frac{\gamma-p-v}{p(\gamma+1)}, \frac{\gamma-1}{2(\gamma+1)}\right\}$ and $\varepsilon$ is a positive constant.

Taking the derivative of $K(t)$ and using Eq.(1.1), we get

$$
\begin{aligned}
K^{\prime}(t) & =(1-\rho) H^{-\rho}(t) H^{\prime}(t)+\varepsilon\left\|u_{t}\right\|^{2}-\varepsilon\|\Delta u\|^{2}+\varepsilon \int_{0}^{t} \mu(t-s) \int_{\Omega} \Delta u(s) \Delta u(t) d x d s \\
& -\varepsilon \int_{\Omega}|u(t)|^{v} u(t) \partial j\left(u_{t}\right)(t) d x+\varepsilon\|u\|_{\gamma+1}^{\gamma+1} \\
& =(1-\rho) H^{-\rho}(t) H^{\prime}(t)+\varepsilon\left\|u_{t}\right\|^{2}-\varepsilon\|\Delta u\|^{2}+\varepsilon \int_{0}^{t} \mu(s) d s\|\Delta u\|^{2}+\varepsilon \int_{0}^{t} \mu(t-s) \int_{\Omega} \Delta u(t)(\Delta u(s)-\Delta u(t)) d x d s \\
& -\varepsilon \int_{\Omega}|u(t)|^{v} u(t) \partial j\left(u_{t}\right)(t) d x+\varepsilon\|u\|_{\gamma+1}^{\gamma+1} .
\end{aligned}
$$

By applying Young's inequality to estimate the fifth term of (3.4) as follows

$$
\left|\int_{0}^{t} \mu(t-s) \int_{\Omega} \Delta u(t)(\Delta u(s)-\Delta u(t)) d x d s\right| \leq \int_{0}^{t} \mu(s) d s\|\Delta u\|^{2}+\frac{1}{4}(\mu \diamond \Delta u)(t) .
$$

From $(A 3)$, since $0<l \leq 1$. Then it follows from the definition of $H(t)$ that

$$
-\|\Delta u\|^{2}=\frac{2}{l} H(t)+\frac{1}{l}\left\|u_{t}\right\|^{2}+\frac{1}{l}(\mu \diamond \Delta u)(t)-\frac{2}{l(\gamma+1)}\|u\|_{\gamma+1}^{\gamma+1} .
$$

Combining (3.4)-(3.6), we obtain

$$
\begin{aligned}
K^{\prime}(t) & \geq(1-\rho) H^{-\rho}(t) H^{\prime}(t)+\varepsilon\left(1+\frac{1}{l}\right)\left\|u_{t}\right\|^{2}+\frac{2}{l} H(t)+\left(\frac{1}{l}-\frac{1}{4}\right)(\mu \diamond \Delta u)(t) \\
& -\varepsilon \int_{\Omega}|u(t)|^{v} u(t) \partial j\left(u_{t}\right)(t) d x+\varepsilon\left(1-\frac{2}{l(\gamma+1)}\right)\|u\|_{\gamma+1}^{\gamma+1} .
\end{aligned}
$$

By assumption $\int_{0}^{t} \mu(\tau) d \tau<\frac{\gamma-1}{\gamma+1}$, we have $1-\frac{2}{l(\gamma+1)}>0$.

In order to estimate fifth term in (3.7), since $q>v+p$, from assumption $(A 1)$ and thanks to Holder's inequality and Young's inequality, we obtain

$$
\begin{aligned}
\left.\left|\int_{\Omega}\right| u(t)\right|^{v} u(t) \partial j\left(u_{t}\right)(t) d x \mid & \leq \int_{\Omega}|u(t)|^{v+1-\frac{v+p+1}{p+1}}|u(t)|^{\frac{v+p+1}{p+1}}\left|u_{t}(t)\right|^{p} d x \\
& \leq C_{0}\left(\int_{\Omega}|u(t)|^{v}\left|u_{t}(t)\right|^{p+1} d x\right)^{\frac{p}{p+1}}\left(\int_{\Omega}|u(t)|^{v+p+1} d x\right)^{\frac{1}{p+1}} \\
& \leq C_{0}|\Omega|^{\frac{\gamma-v-p}{\gamma+1}}\left(\int_{\Omega}|u(t)|^{v}\left|u_{t}(t)\right|^{p+1} d x\right)^{\frac{p}{p+1}}\|u(t)\|_{\gamma+1}^{\frac{v+p+1}{p+1}} \\
& \leq \beta\left(H^{\prime}(t)\right)^{\frac{p}{p+1}}\|u(t)\|_{\gamma+1}^{\frac{v+p+1}{p+1}} \\
& \leq \beta\left(\delta^{-\frac{1}{p}} H^{\prime}(t)+\delta\|u(t)\|_{\gamma+1}^{v+p+1}\right)
\end{aligned}
$$


where constant $\delta>0$ is specified later and $\beta=C_{0} C_{1}^{-\frac{p}{p+1}}|\Omega|^{\frac{\gamma-v-p}{\gamma+1}}$.

Hence, (3.7) becomes

$$
\begin{aligned}
K^{\prime}(t) & \geq\left[(1-\rho) H^{-\rho}(t)-\varepsilon \beta \delta^{-\frac{1}{p}}\right] H^{\prime}(t)+\varepsilon\left(1+\frac{1}{l}\right)\left\|u_{t}\right\|^{2}+\varepsilon \frac{2}{l} H(t)+\varepsilon\left(\frac{1}{l}-\frac{1}{4}\right)(\mu \diamond \Delta u)(t) \\
& +\varepsilon\left(1-\frac{2}{l(\gamma+1)}\right)\|u\|_{\gamma+1}^{\gamma+1}-\varepsilon \beta \delta\|u(t)\|_{\gamma+1}^{v+p+1} .
\end{aligned}
$$

The choice of $\delta\left(\right.$ i.e. $\left.\delta=\frac{1}{\beta}\left(\frac{1}{2}-\frac{1}{l(\gamma+1)}\right)\|u\|_{\gamma+1}^{\gamma-v-p}\right)$, then

$$
\varepsilon \beta \delta\|u(t)\|_{\gamma+1}^{\gamma+p+1}=\varepsilon\left(\frac{1}{2}-\frac{1}{l(\gamma+1)}\right)\|u\|_{\gamma+1}^{\gamma+1} .
$$

Furthermore, since $\|u\|_{\gamma+1} \geq[(\gamma+1) H(0)]^{\frac{1}{\gamma+1}}$ by (3.3) and $v+p-\gamma+p(\gamma+1) \rho \leq 0$, then

$$
\begin{aligned}
(1-\rho) H^{-\rho}(t)-\varepsilon \beta \delta^{-\frac{1}{p}} & =H^{-\rho}(t)\left[1-\rho-\varepsilon \beta \delta^{-\frac{1}{p}} H^{\rho}(t)\right] \\
& \geq H^{-\rho}(t)\left[1-\rho-\varepsilon \beta^{1+\frac{1}{p}}\left(\frac{1}{2}-\frac{1}{l(\gamma+1)}\right)^{-\frac{1}{p}}(\gamma+1)^{-\rho}\|u\|_{\gamma+1}^{\frac{p+v-\gamma+p(\gamma+1) \rho}{p}}\right] \\
& \geq H^{-\rho}(t)\left[1-\rho-\varepsilon \beta^{1+\frac{1}{p}}\left(\frac{1}{2}-\frac{1}{l(\gamma+1)}\right)^{-\frac{1}{p}}(\gamma+1)^{-\rho-\frac{q-p-v}{p(q+1)}} H(0)_{\gamma+1}^{\rho-\frac{\gamma-v-p}{p(\gamma+1)}}\right] \\
& \geq H^{-\rho}(t)\left[1-\rho-\varepsilon \beta^{1+\frac{1}{p}} \chi\right]
\end{aligned}
$$

where $\chi=\left(\frac{1}{2}-\frac{1}{l(\gamma+1)}\right)^{-\frac{1}{p}}(\gamma+1)^{\rho-\frac{q-p-v}{p(q+1)}} H(0)_{\gamma+1}^{\rho-\frac{\gamma-v-p}{p(\gamma+1)}}$. Now, we choose $\varepsilon$ to be sufficiently small such that

$$
1-\rho-\varepsilon \beta^{1+\frac{1}{p}} \chi>0 .
$$

Then (3.10) and (3.9) yield

$$
K^{\prime}(t) \geq \varepsilon C\left[H(t)+\left\|u_{t}(t)\right\|^{2}+\|u\|_{\gamma+1}^{\gamma+1}+(\mu \diamond \Delta u)(t)\right]
$$

where $C>0$ is a constant that does not depended on $\varepsilon$. Especially, (3.11) means that $K(t)$ is increasing on $[0, T)$, with

$$
K(t)=H^{1-\rho}(t)+\varepsilon \int_{\Omega} u u_{t} d x \geq H^{1-\rho}(0)+\varepsilon \int_{\Omega} u_{0} u_{1} d x .
$$

We also select $\varepsilon$ to be sufficiently small such that $K(0)>0$, thus $K(t) \geq K(0)>0$ for $t \geq 0$.

Let $\eta=\frac{1}{1-\rho}$. Since $0<\rho<\frac{1}{2}$, it is evident that $2>\eta>1$. By using the following inequality

$$
|x+y|^{\eta} \leq 2^{\eta-1}\left(|x|^{\eta}+|y|^{\eta}\right) \text { for } \eta \geq 1
$$

applying Young's inequality, we get

$$
\begin{aligned}
K^{\eta}(t) & \leq 2^{\eta-1}\left(H(t)+\varepsilon\|u(t)\|^{\eta}\left\|u_{t}(t)\right\|^{\eta}\right) \\
& \leq C\left(H(t)+\left\|u_{t}(t)\right\|^{2}+\|u(t)\|_{\gamma+1}^{\frac{1}{\frac{1}{2}-\rho}}\right) .
\end{aligned}
$$

By the choice of $\rho$, we have $\frac{1}{2}-\rho>\frac{1}{\gamma+1}$. Now applying the inequality

$$
a^{\sigma} \leq\left(1+\frac{1}{b}\right)(b+a), a \geq 0,0 \leq \sigma \leq 1, b>0,
$$

and taking $a=\|u(t)\|_{\gamma+1}^{\gamma+1}, \eta=\frac{1}{\left(\frac{1}{2}-\rho\right)(\gamma+1)}<1$, and $b=H(0)$, we obtain

$$
\begin{aligned}
\|u(t)\|_{\gamma+1}^{\frac{1}{\frac{1}{2}-\rho}} & \leq\left(1+\frac{1}{H(0)}\right)\left(H(0)+\|u(t)\|_{\gamma+1}^{\gamma+1}\right) \\
& \leq C\left(H(t)+\|u(t)\|_{\gamma+1}^{\gamma+1}\right) .
\end{aligned}
$$

Therefore, by combining of (3.12) and (3.13), we obatin

$$
\begin{aligned}
K^{\eta}(t) & \leq C\left(H(t)+\left\|u_{t}(t)\right\|^{2}+\|u(t)\|_{\gamma+1}^{\gamma+1}\right) \\
& \leq C\left(H(t)+\left\|u_{t}(t)\right\|^{2}+\|u(t)\|_{\gamma+1}^{\gamma+1}+(\mu \diamond \Delta u)(t)\right) .
\end{aligned}
$$

Thus, (3.11) and (3.14) arrive at

$$
K^{\prime}(t) \geq C K^{\eta}(t), t \in[0, T] .
$$

In the end, from (3.15) and $\eta=\frac{1}{1-\rho}>1$, we see that $K(t)=H^{1-\rho}(t)+\varepsilon \int_{\Omega} u u_{t} d x$ blow up in finite time. This completes the proof. 


\section{Growth}

In this section, we goal to show that the energy grow up as an exponential function as time as goes to infinity.

Theorem 4.1. Let (A1)-(A4) hold. Assume further that $\gamma>v+p$ and $E(0)<0$ and $u$ be a any solution to (1.1) grows exponentially.

Proof. We define

$$
Z(t)=H(t)+\varepsilon \int_{\Omega} u u_{t} d x
$$

where $H(t)=-E(t)$ and $0<\varepsilon \leq 1$. By derivating (4.1) and using Eq.(1.1), we have

$$
\begin{aligned}
Z^{\prime}(t) & =H^{\prime}(t)+\varepsilon\left\|u_{t}\right\|^{2}-\varepsilon\|\Delta u\|^{2}+\varepsilon \int_{0}^{t} \mu(t-s) \int_{\Omega} \Delta u(s) \Delta u(t) d x d s-\varepsilon \int_{\Omega}|u(t)|^{v} u(t) \partial j\left(u_{t}\right)(t) d x+\varepsilon\|u\|_{\gamma+1}^{\gamma+1} \\
& =H^{\prime}(t)+\varepsilon\left\|u_{t}\right\|^{2}-\varepsilon\|\Delta u\|^{2}+\varepsilon \int_{0}^{t} \mu(s) d s\|\Delta u\|^{2}+\varepsilon \int_{0}^{t} \mu(t-s) \int_{\Omega} \Delta u(t)(\Delta u(s)-\Delta u(t)) d x d s \\
& -\varepsilon \int_{\Omega}|u(t)|^{v} u(t) \partial j\left(u_{t}\right)(t) d x+\varepsilon\|u\|_{\gamma+1}^{\gamma+1} .
\end{aligned}
$$

By using (3.5), the assumption $(A 3)$ and the definition $H(t)$, we have $0<l \leq 1$ and

$$
Z^{\prime}(t) \geq H^{\prime}(t)+\varepsilon\left(1+\frac{1}{l}\right)\left\|u_{t}\right\|^{2}+\frac{2}{l} H(t)+\left(\frac{1}{l}-\frac{1}{4}\right)(\mu \diamond \Delta u)(t)-\varepsilon \int_{\Omega}|u(t)|^{v} u(t) \partial j\left(u_{t}\right)(t) d x+\varepsilon\left(1-\frac{2}{l(\gamma+1)}\right)\|u\|_{\gamma+1}^{\gamma+1} .
$$

By the assumption $\int_{0}^{t} \mu(\tau) d \tau<\frac{\gamma-1}{\gamma+1}$ and using (3.8), we get

$$
\begin{aligned}
Z^{\prime}(t) & \geq\left[1-\varepsilon \beta \delta^{-\frac{1}{p}}\right] H^{\prime}(t)+\varepsilon\left(1+\frac{1}{l}\right)\left\|u_{t}\right\|^{2}+\varepsilon \frac{2}{l} H(t)+\varepsilon\left(\frac{1}{l}-\frac{1}{4}\right)(\mu \diamond \Delta u)(t) \\
& +\varepsilon\left(1-\frac{2}{l(\gamma+1)}\right)\|u\|_{\gamma+1}^{\gamma+1}-\varepsilon \beta \delta\|u(t)\|_{\gamma+1}^{v+p+1} .
\end{aligned}
$$

The choice of $\delta$ (i.e. $\left.\delta=\frac{1}{\beta}\left(\frac{1}{2}-\frac{1}{l(\gamma+1)}\right)\|u\|_{\gamma+1}^{\gamma-v-p}\right)$, then

$$
\varepsilon \beta \delta\|u(t)\|_{\gamma+1}^{v+p+1}=\varepsilon\left(\frac{1}{2}-\frac{1}{l(\gamma+1)}\right)\|u\|_{\gamma+1}^{\gamma+1}
$$

Furthermore, since $\|u\|_{\gamma+1} \geq[(\gamma+1) H(0)]^{\frac{1}{\gamma+1}}$ by (3.3) and assumption $v+p-\gamma \leq 0$, then

$$
\begin{aligned}
1-\varepsilon \beta \delta^{-\frac{1}{p}} & \geq 1-\varepsilon \beta^{1+\frac{1}{p}}\left(\frac{1}{2}-\frac{1}{l(\gamma+1)}\right)^{-\frac{1}{p}}(\gamma+1)^{-\frac{\gamma-p-v}{p(\gamma+1)}} H(0)_{\gamma+1}^{-\frac{\gamma-v-p}{p(\gamma+1)}} \\
& \geq 1-\varepsilon \beta^{1+\frac{1}{p}} P
\end{aligned}
$$

where $P=\left(\frac{1}{2}-\frac{1}{l(\gamma+1)}\right)^{-\frac{1}{p}}(\gamma+1)^{-\frac{\gamma-p-v}{p(\gamma+1)}} H(0)_{\gamma+1}^{-\frac{\gamma-v-p}{p(\gamma+1)}}$. Now, we choose $\varepsilon$ to be sufficiently small such that

$$
1-\varepsilon \beta^{1+\frac{1}{p}} P>0
$$

Thus,

$$
Z^{\prime}(t) \geq \varepsilon C\left[H(t)+\left\|u_{t}(t)\right\|^{2}+\|u\|_{\gamma+1}^{\gamma+1}+(\mu \diamond \Delta u)(t)\right]
$$

where $C>0$ is a constant that does not depended on $\varepsilon$.

Now, applying Young's inequality, and Sobolev Poincare inequality we have

$$
\begin{aligned}
Z(t) & \leq H(t)+\varepsilon\|u\|\left\|u_{t}\right\| \\
& \leq C\left(H(t)+\left\|u_{t}\right\|^{2}+\|u\|^{2}\right) .
\end{aligned}
$$

Now, in order the estimate $\|u\|^{2}$ term we apply the inequality $a^{l} \leq(a+1) \leq\left(1+\frac{1}{b}\right)(a+b)$ for $a=\|u\|_{\gamma+1}^{\gamma+1}, l=2 / \gamma+1<1, b=H(0)$, we have

$$
\begin{aligned}
\|u\|^{2} & \leq C\|u\|_{\gamma+1}^{2} \\
& =C\left(\|u\|_{\gamma+1}^{\gamma+1}\right)^{\frac{2}{\gamma+1}} \\
& \leq\left(1+\frac{1}{H(0)}\right)\left(\|u\|_{\gamma+1}^{\gamma+1}+H(0)\right) \\
& \leq C\left(\|u\|_{\gamma+1}^{\gamma+1}+H(t)\right) .
\end{aligned}
$$


Thus,

$$
Z(t) \leq C\left[H(t)+\left\|u_{t}(t)\right\|^{2}+\|u\|_{\gamma+1}^{\gamma+1}+(\mu \diamond \Delta u)(t)\right] .
$$

Therefore, (4.5) and (4.7) arrive at

$$
Z^{\prime}(t) \geq \xi Z(t), \quad t \geq 0
$$

This completes the proof.

\section{Conclusion}

In this work, we obtained the finite time bolw up and growth of solutions for a Petrovsky equation with degenerate damping in a bounded domain. This improves and extends many results in the literature.

\section{Acknowledgements}

The authors would like to express their sincere thanks to the editor and the anonymous reviewers for their helpful comments and suggestions.

\section{Funding}

There is no funding for this work.

\section{Availability of data and materials}

Not applicable.

\section{Competing interests}

The authors declare that they have no competing interests.

\section{Author's contributions}

All authors contributed equally to the writing of this paper. All authors read and approved the final manuscript.

\section{References}

[1] V. Barbu, I. Lasiecka, M. A. Rammaha, Existence and uniqueness of solutions to wave equations with nonlinear degenerate damping and source terms, Control Cybernetics, 34(3) (2005), 665-687.

[2] J. M. Rivera, E. C. Lapa, R. Barreto, Decay rates for viscoelastic plates with memory, J. Elasticity, 44(1) (1996), 61-87.

[3] F. Alabau-Boussouira, P. Cannarsa, D. Sforza, Decay estimates for the second order evolution equation with memory, J. Func. Anal., 245(5) (2008), 1342-1372.

[4] F. Tahamtani, M. Shahrouzi, Existence and blow up of solutions to a Petrovsky equation with memory and nonlinear source term, Bound. Value Probl., 50(2012) (2012), 1-15.

[5] F. Li, Q. Gao, Blow-up of solution for a nonlinear Petrovsky type equation with memory, Appl. Math. Comput., 274 (2016), $383-392$.

[6] L. Liu, F. Sun, Y. Wu, Blow-up of solutions for a nonlinear Petrovsky type equation with initial data at arbitrary high energy level, Bound. Value Probl., 15(2019) (2019), 1-18.

[7] L. Liu, F. Sun, Y. Wu, Finite time blow-up for a nonlinear viscoelastic Petrovsky equation with high initial energy, SN Partial Differ. Equ. Appl., 1(31) (2020), 1-18.

[8] S. A. Messaoudi, Global existence and nonexistence in a system of Petrovsky, J.Math. Anal. Appl., 265(2) (2002), 296-308.

[9] W. Chen,Y. Zhou, Global nonexistence for a semilinear Petrovsky equation, Nonlinear Anal., 70 (2009), 3203-3208.

[10] E. Pişkin, N. Polat, On the Decay of Solutions for a Nonlinear Petrovsky Equation, Math. Sci. Letter, 3(1) (2013), $43-47$.

[11] S. Antontsev, J. Ferreira, E. Pişkin, Existence and blow up of solutions for a strongly damped Petrovsky equation with variable-exponent nonlinearities, Electron. J. Diff. Eq., 2021(6) (2021), 1-18.

[12] E. Piskin, T. Uysal, Blow up of the solutions for the Petrovsky equation with fractional damping terms, Malaya J. Math., 6(1) (2018), 85-90.

[13] E. Piskin, Z. Çalışır, Decay and blow up at infinite time of solutions for a logarithmic Petrovsky equation, Tbilisi Math. J., 13(4) (2020), 113-127.

[14] H. Yüksekkaya, E. Pişkin, Blow up of Solutions for Petrovsky Equation with Delay term, J. Nepal Math. Soc., 4(1) (2021), 76-84.

[15] H. A. Levine, J. Serrin, Global nonexistence theorems for quasilinear evolution with dissipation, Arch. Ration. Mech. Anal., 137 (1997), 341-361.

[16] D. R. Pitts, M. A. Rammaha, Global existence and nonexistence theorems for nonlinear wave equations, Indiana Uni. Math. J., 51(6) (2002), 1479-1509.

[17] V. Barbu, I. Lasiecka, M. A. Rammaha, Blow-up of generalized solutions to wave equations with nonlinear degenerate damping and source terms, IIndiana Uni. Math. J.,, 56(3) (2007), 995-1022.

[18] V. Barbu, I. Lasiecka, M. A. Rammaha, On nonlinear wave equations with degenerate damping and source terms, Trans. Amer. Math. Soc., 357(7) (2005), 2571-2611

[19] Q. Hu, H. Zhang, Blow up and asymptotic stability of weak solutions to wave equations with nonlinear degenerate damping and source terms, Electron. J. Diff. Eq., 2007(76) (2007), 1-10.

[20] S. Xiao, W. Shubin, A blow-up result with arbitrary positive initial energy for nonlinear wave equations with degenerate damping terms, J. Diff. Eq., 32 (2019), 181-190.

[21] F. Ekinci, E. Pişkin, Nonexistence of global solutions for the Timoshenko equation with degenerate damping, Discovering Mathematics (Menemui Matematik), 43(1) (2021), 1-8. 